\title{
Clinical Trial Data
}

National Cancer Institute

\section{Source}

National Cancer Institute. Clinical Trial Data. NCI Thesaurus. Code C142441.

Information collected during the course of a scientifically-controlled study of the safety and efficacy of a therapeutic agent, such as a drug or vaccine, using consenting human subjects. 